\title{
FABRICATION OF POLY(LACTIC ACID) - POLY(ETHYLENE OXIDE) ELECTROSPUN MEMBRANES WITH CONTROLLED MICRO TO NANOFIBER SIZES
}

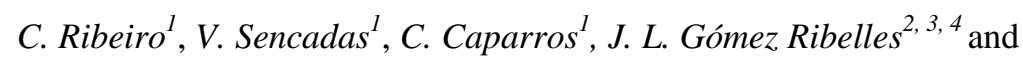 \\ S. Lanceros-Méndez,"*
}

${ }^{1}$ Centro/Departamento de Física da Universidade do Minho,Campus de Gualtar, 4710-
057 Braga, Portugal
${ }^{2}$ Centro de Biomateriales e Ingeniería Tisular, Universidad Politécnica de Valencia,
46022 Valencia, Spain
${ }^{3}$ Centro de Investigación Príncipe Felipe, Autopista del Saler 16, 46013 Valencia, Spain
${ }^{4}$ CIBER en Bioingeniería, Biomateriales y Nanomedicina, Valencia, Spain

[*] Corresponding author: lanceros@ fisica.uminho.pt

\begin{abstract}
Biodegradable poly(L-lactide acid) (PLLA) nanofiber membranes were prepared by electrospinning of PLLA and poly(ethylene oxide) (PEO). The selective removal of PEO by water allows to obtain smaller fiber diameters and to increase the porosity of the membranes in comparison to PLLA membranes obtained under the same electrospinning conditions. After removal of PEO membranes with fiber sizes of 260 $\mathrm{nm}$ and average porosity close to $80 \%$ are obtained. Thermal and infrared results confirm the poor miscibility of PLLA and PEO, with the PEO randomly distributed along the PLLA fibers. On the other, PLLA and PEO mixing strongly affect their respective degradation temperatures. The influence of the PEO in the electrospinning process is discussed and the results are correlated to the evolution of the PLLA fiber diameter.
\end{abstract}

Formatada: Não Realce Formatada: Não Realce

Formatada: Não Realce

Formatada: Não Realce

Keywords: electrospinning; membranes; nanofibers; biomaterials. 


\section{Introduction}

When the average diameter of polymer fibers are shrunk from micrometers to nanometers, there appear several interesting characteristics such as larger surface area to volume ratio, flexibility in surface functionalities and superior mechanical properties, when compared to any other known form of the material [1-2].

Código de campo alterado

It has been found that morphological characteristics such as fiber diameter and the uniformity of the electrospun polymer fibers are dependent on parameters such as processing conditions, solution properties and environmental conditions [2].

Código de campo alterado

Electrospinning has proven to be an excellent method for the synthesis of submicron- or nano-fibers from polymer solutions for a wide range of polymeric materials. The electrospinning process was described by Formhals at the beginning of the 1930s, towards the commercialization of textiles yarns [3]. This technique is relatively versatile, simple, fast and efficient. Electrospinning membranes have attracted interest to be used in biomedical applications, such as scaffolds for tissue engineering, sutures, implants and controlled drug delivery systems, but also in other applications including filtration, sensors, batteries, cell phones and for chemical warfare protection, among others. [1, 4-5]. In all these applications fiber diameter and membrane porosity are among the key factors defining membrane performance.

Several processing parameters can influence the morphology and properties of the electrospun fibers. The most important ones are those corresponding to the initial polymer solution, the solution concentration and the molecular weight of the polymer [1]. Moreover, the parameters that control the jet formation and solvent evaporation are the flow rate through the needle, the needle diameter, distance from the tip to the collector, temperature, applied voltage, and the collection procedure, static or dynamic, using a rotating drum collector [6].

Poly(L-lactic acid) (PLLA) is a biodegradable polyester that has been used as a biomaterial for temporary therapeutic applications, controlled drug release, support for cell culture and tissue engineering. Its degradation by simple hydrolysis of the ester backbone in aqueous environments such as body fluids makes it a good candidate for the implementation of temporary applications.

There are several works showing the effect of the electrospinning processing parameters on the morphology of PLLA fibers [7-10]. Tsuji et al. found that the fiber diameter decreases with increasing applied voltage [11]. Tomaszewski et al. studied the effect of

Formatada: Não Realce

Código de campo alterado

Formatada: Não Realce

Código de campo alterado

Código de campo alterado

Formatada: Não Realce

Formatada: Não Realce

Código de campo alterado 
poly(L-lactide) molecular weight and viscosity of spinning solutions in fiber thickness [12]. $\mathrm{Gu}$ and Ren investigated the influence of applied voltage and polymer concentration on fiber diameter, and found that fiber diameter tended to increase with increasing polymer concentration and decreases with increasing applied voltage [13]. The influence of solution viscosity and electrical conductivity on fiber diameter and morphology was also investigated [14]. It was found that by reducing the PLLA concentrations in the solution the fiber diameter decreases but, on the other hand, the formation of beaded fibers was observed. The formation of beads can be reduced nevertheless by increasing the electrical conductivity of the solution.

Despite these efforts, the precise knowledge for controlling the electrospinning parameters in order to obtain fibers with the desired morphology, crystallinity and molecular structure is still lacking, in particular in the case that nanosize fibers are to be obtained.

The processing of an inorganic nanocomponent or polymer into another kind of polymer or inorganic matrix by using electrospinning has been also studied by several authors [10]. By co-electrospinning of two components that can be dissolved in a common solvent, composite nanofibers can be obtained after the solvent evaporation. The basic idea is to synthesize composite nanofibers allowing to provide new properties to the composite materials or to control the fiber morphology [10]. Several studies prove that it is possible to control the morphology of the composite nanofibers by proper understanding the electrospinning process. As an example, Jing and coworkers prove to obtain core/shell nanofibers of PEO/PEG-PLA by electrospinning [15]. It was observed that the polymer with higher viscosity moves into the center of the electrospun fibers and the component with lower viscosity is located outside. Additionally, as solvent evaporation is fast, the formation of core/shell fibers occurs in blend system with high molecular mobility. In this way, polymer blends with lower molecular weight tend to form core/shell fibers rather than a co-continuous structure, as a result of their higher molecular mobility [16].

Poly(ethylene oxide) (PEO) is a semi-crystalline hydrophilic polymer with high potential for biomedical applications, due to its good biocompatibility and low toxicity. Miscibility and phase separation of PLLA and PEO have been studied by several authors $[7,9,17]$. It was found that melting point reduction of both PLLA and PEO polymers occurs, in particular at PEO contents below 0.2 weight fraction. It was also suggested that the inclusion of the two polymer molecules occurs in the amorphous state
Código de campo alterado

\begin{tabular}{|l|}
\hline Formatada: Não Realce \\
\hline Formatada: Não Realce \\
\hline Código de campo alterado \\
\hline Código de campo alterado \\
\hline Formatada: Não Realce \\
\hline Formatada: Não Realce \\
\hline Formatada: Não Realce \\
\hline Formatada: Não Realce \\
\hline
\end{tabular}

Formatada: Não Realce

Formatada: Não Realce

Código de campo alterado

Formatada: Não Realce

Formatada: Não Realce

Código de campo alterado

Formatada: Não Realce

Código de campo alterado

Formatada: Não Realce

Formatada: Não Realce

Formatada: Não Realce

Código de campo alterado

Código de campo alterado 
[9]. Nakafaku studied the melting and crystallization behavior under pressure, assuming its semi-miscible behavior. However, it is claimed that the melting and crystallization behavior under different synthesization parameters is different depending on the molecular weight of the PLLA and PEO [18].

Although the influence of the parameters of the electrospinning process on fiber diameter has been well analyzed in PLLA, the information on the conditions required to obtain ultra-fine nanofibers is scarce yet. In this work, PLLA and PEO blends were prepared by electrospinning in order to achieve PLLA membranes with nanosize fibers. The influence of the PEO in the blend electrospinning process is discussed, and the results are correlated to the evolution of the PLLA fiber diameter. The selective removal of PEO by water gives origin to smaller fiber diameter and to increased porosity of the membranes in comparison to the PLLA membranes obtained under the same electrospinning conditions. This study shows therefore an easy route to obtain nanosized fiber PLLA porous membranes with large application potential in the biomedical field.

\section{Experimental}

Materials: Poly(L-lactic acid) (PLLA) with a average molecular weight of 217.000 $225.000 \mathrm{~g} / \mathrm{mol}$, Purasorb PL18 purchased from Purac (Netherlands) and Polyethylene oxide (PEO) with an average molecular weight of $100.000 \mathrm{~g} / \mathrm{mol}$ supplied by Polysciences (USA) were dissolved in a mixture of $\mathrm{N}, \mathrm{N}$-dimethylformamide (DMF, from Merck) and dichloromethane (MC, from Sigma-Aldrich) (3/7 vol/vol) to achieve a polymer concentration of $10 \mathrm{wt} \%$ of the total solution. The PLLA/PEO blends used in this work contained $75 \%$ and $50 \%$ by weight respectively of PLLA. The polymer blend was dissolved at room temperature in a magnetic stirrer until complete polymer dissolution.

Electrospinning: The polymer solution was placed in a commercial plastic syringe (10 ml) fitted with a steel needle with $500 \mu \mathrm{m}$ of inner diameter. Electrospinning was conducted at $20 \mathrm{kV}$ with a high voltage power supply from Glassman (model PS/FC30P04). A syringe pump (from Syringepump) was used to feed the polymer 
solution into the needle tip at rate between $2 \mathrm{ml}^{-1}$. The electrospun fibers were collected in a ground collecting plate (random fibers), placed at $15 \mathrm{~cm}$ from the needle.

Characterization: Electrospun fiber samples after and before PEO removal in water were coated with a thin gold layer using a sputter coating (Polaron, model SC502 sputter coater) and their morphology was analyzed using a scanning electron microscopy (SEM) (model JSM-6300, JEOL) with an accelerating voltage of $5 \mathrm{kV}$. The nanofibers average diameter and its distribution was calculated over approximately 50 fibers using de SEM image (5000X magnification) and the Image J software [19]. A scanning probe microscope (Model 5500 from Agilent) was employed for the AFM analysis of the samples before and after PEO removal.

Infrared measurements (FTIR) were performed at room temperature in a Perkin-Elmer Spectrum 100 apparatus in ATR mode from 4000 to $650 \mathrm{~cm}^{-1}$. FTIR spectra were collected with 32 scans and a resolution of $4 \mathrm{~cm}^{-1}$.

The thermal behavior of the electrospun fiber mats was analyzed by differential scanning calorimetry (DSC) measurements with a Perkin Elmer Diamond DSC apparatus. The samples were cut into small pieces from the middle region of the electrospun membranes, placed into $40 \mu \mathrm{l}$ aluminum pans and heated between 30 and $200{ }^{\circ} \mathrm{C}$ at a heating rate of $10^{\circ} \mathrm{C} \cdot \mathrm{min}^{-1}$. All experiments were performed under a nitrogen purge. The glass transition temperature $\left(T_{g}\right)$, cold-crystallization temperature $\left(T_{C C}\right)$, melting temperature $\left(T_{m}\right)$, cold-crystallization enthalpy $\left(\Delta H_{C C}\right)$, melting enthalpy $\left(\Delta H_{m}\right)$ and degree of crystallinity $\left(\Delta X_{C}\right)$ of all electrospun samples were obtained.

Thermogravimetric analysis (TGA) was carried out in a Perkin-Elmer Pyris-1 TGA apparatus from 30 to $700{ }^{\circ} \mathrm{C}$ at $20^{\circ} \mathrm{C} \cdot \mathrm{min}^{-1}$ under a nitrogen atmosphere.

Due to the porous nature of the membrane, which is fully opened and interconnected, a suitable method to estimate the porosity of the samples is the pycnometer test. In this work, and due to nature of the polymers used (PEO is water soluble), the porosity of the samples were measured by an improved weight-method. The weight of the pycnometer, filled with ethanol, was weighted and labeled as W1; the sample, whose weight was Ws, was immersed in ethanol. After the pores of the membranes were saturated by ethanol, additional ethanol was added to complete the volume of the pycnometer. Then, the pycnometer was weighted and labeled as W2 and the sample filled with ethanol was
Formatada: Não Realce

Código de campo alterado

Formatada: Não Realce

Formatada: Não Realce

Formatada: Não Realce 
taken out of the pycnometer. The residual weight of the ethanol and the pycnometer was labeled W3. The porosity of the scaffold was calculated according to $\varepsilon=\frac{W_{2}-W_{3}-W_{S}}{W_{1}-W_{3}}$. The porosity of each membrane was obtained as the average of the values determined in three samples.

\section{Results and discussion}

\subsection{Scanning Electron Microscopy}

Electrospinning of PLLA generally results in fibers with diameters in the range between $0.6-6.0 \mu \mathrm{m}$, depending on the variation of the selected processing parameters [20, 21]. Ultra-fine nanofibers with average diameter lower than $100 \mathrm{~nm}$ are obtained using a mixture of solvents like dichloromethane/pyridine [2].

A quite high number of parameters have influence in the electrospinning process and any study must fix some of them [1, 22]. In this work, solutions of PLLA and PEO in a blended solution of MC and DMF has been used. The high polarity of the DMF facilitates the fiber formation while the high volatility of the MC allows a quick evaporation from the fiber, maintaining theintegrity of the fiber during the traveling from the tip of the needle to the target.

Once the PLLA-PEO membranes are obtained, PEO was removed by immersing the membranes in deionized water. After 24 hours, the samples were dried at $40{ }^{\circ} \mathrm{C}$ until they reach a constant mass. The PEO was in this way fully removed from the PLLAPEO electrospun fiber mesh, which is accompanied by a mass reduction of 25 and $50 \%$ of the membrane, respectively. Figure 1 shows the SEM images of the obtained membrane for the PLLA-PEO samples with 50 and $75 \%$ of PLLA, before the PEO removal, and the respective fiber diameter distribution. It is observed that all fibrils present a very smooth surface and that the obtained membranes are very porous.
Formatada: Não Realce

Formatada: Não Realce

Código de campo alterado

Código de campo alterado

Código de campo alterado

Formatada: Não Realce

Formatada: Não Realce

Formatada: Não Realce

Formatada: Não Realce

Formatada: Não Realce

Formatada: Não Realce

Formatada: Não Realce 


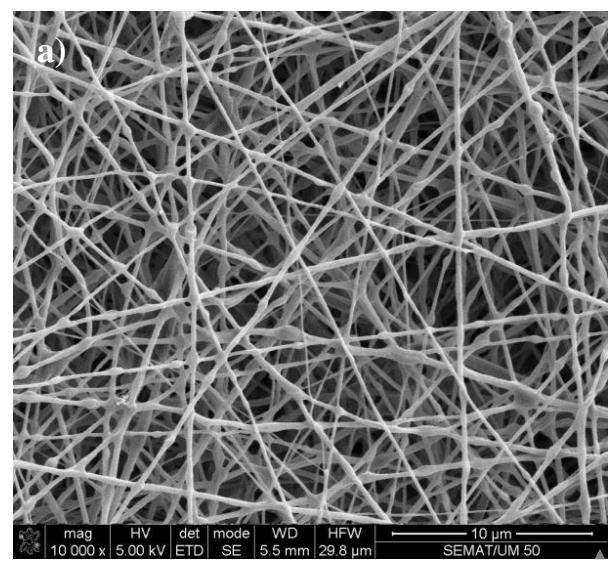

b)

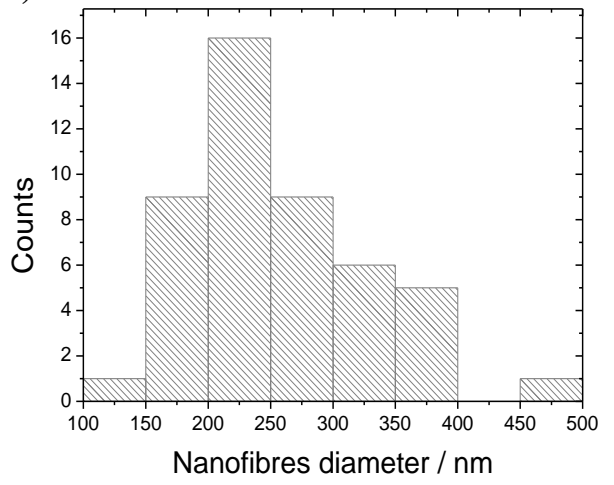

Código de campo alterado

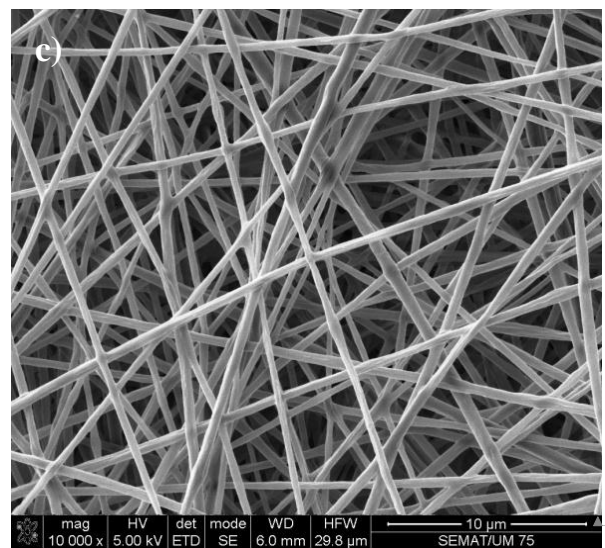

d)

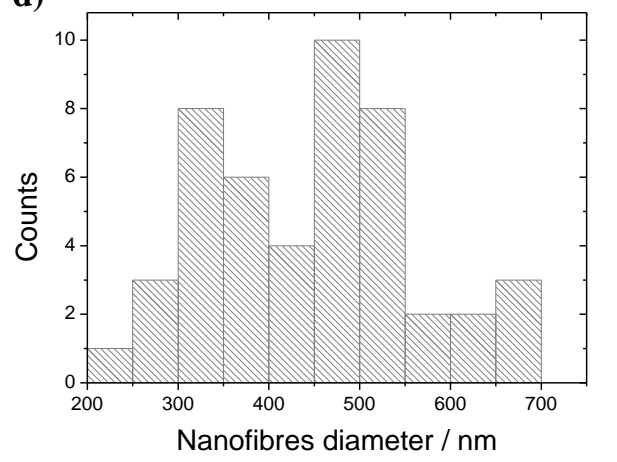

Código de campo alterado

Figure 1 - Morphology of the PLLA-PEO membranes before PEO removal: a) SEM image of the sample with 50\% of PLLA, b) Fiber distribution for the 50/50 PLLA-PEO membrane, c) SEM image of the sample with $75 \%$ of PLLA sample, d) Fiber distribution for the 75/25 PLLA-PEO scaffold.

From figure 1, it is possible to observe that the amount of PEO in the sample strongly influences the fiber distribution in the electrospun mesh. For the sample with $75 \%$ of PLLA and $25 \%$ of PEO, the fiber average diameter was $447 \pm 115 \mathrm{~nm}$ (figure $1 \mathrm{c}$ and d), but for the sample with 50\% of PLLA and 50\% of PEO a reduction of the fiber average diameter to $262 \pm 70 \mathrm{~nm}$ (figure 1a and b) was observed. The samples obtained under the same processing conditions for pure PLLA sample has an average fiber diameter of $1000 \mathrm{~nm} \pm 300 \mathrm{~nm}$, as can be observed in figure 2 . 

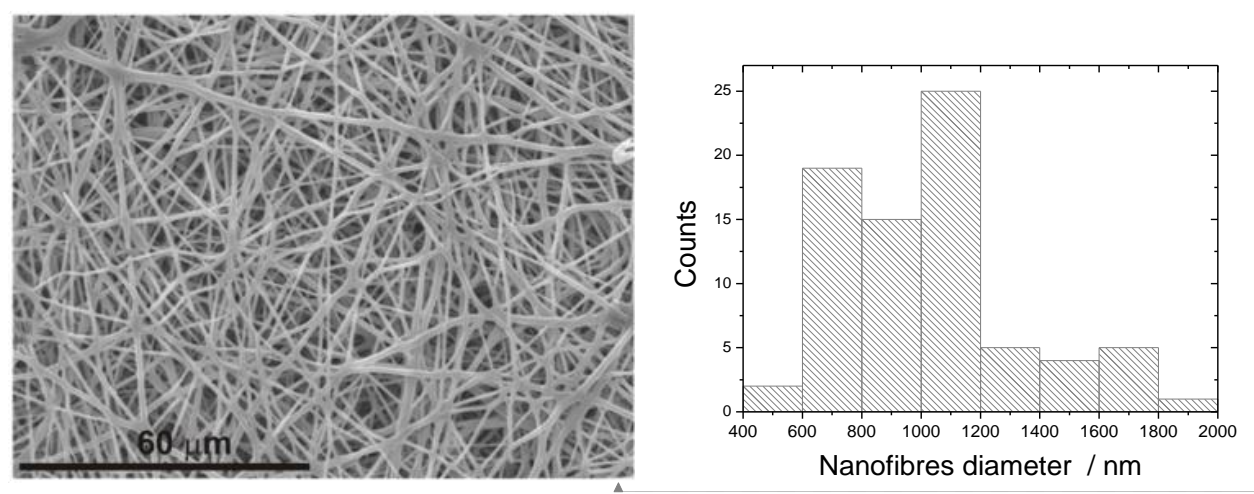

Figure 2 - PLLA membrane obtained for the pure polymer by electrospinning at 20 $\mathrm{kV}$ with a $0.5 \mathrm{~mm}$ inner diameter needle placed at $15 \mathrm{~cm}$ from the collector.

This decrease of the nanofiber diameter with increasing PEO leads to the conclusion that the critical electric field needed to start the Taylor cone and consequently the polymer jet for the PLLA and PEO are not the same, being smaller for the PEO polymer. Therefore, the influence of the applied voltage on both polymer chains is not the same.

The electric field is the drive motor to the jet initiation. In general, once the electric field is applied on the droplet of the polymer solution at the tip of the spinneret, the liquid surface becomes charged due to the motion of the ions through the liquid. When the electric field is high enough such that the electric force overcomes the forces associated to the surface tension, a quasi-stable, straight and electrically charged jet is ejected $[1$, 22]. The balance between the surface tension and the electric force is critical to determine the initial cone shape of the polymer solution at the needle tip. It was observed in our samples that the diameter of the nanofibers for the solution with PLLA polymer is larger than for the PLLA-PEO blend solution.

The mechanisms behind the electrospinning technique are not well understood in detail so far and some intuitive conjectures are given as follows to explain the observed facts. In the case of the PLLA-PEO electrospun nanofibers, the critical electric field at which the repulsive electrostatic forces overcome the surface tension are not the same for both polymers. The discharge polymer solution undergoes instability and elongation process, which allows the jet to become very long and thin and, in this stage, is the elongation of the PEO in the solution that stretches the PLLA present in the solution, giving origin to PLLA nanofibers with smaller diameter for the membranes with higher PEO content (figures 1 and 2). 
Another explanation for the significant reduction on the fiber diameter is related to the viscosity of the solution that decreases in the presence of the PEO [7, 15].

After removal of the PEO from the polymer mesh by immersing the scaffold in water, the fiber distribution, morphology and geometry was characterized by SEM, and the results are shown in figure 3.

Formatada: Não Realce

It was observed that for the case of the PLLA-PEO electrospun polymer blend the average diameter of the nanofibers decrease in both cases after removal of the PEO, being more pronounced for the 75/25 PLLA-PEO polymer blend. The average diameter found for the samples with 75/25 of PLLA-PEO suffer a reduction from $447 \pm 115 \mathrm{~nm}$ to $353 \pm 80 \mathrm{~nm}$, and for the 50/50 PLLA-PEO, that reduction was not so pronounced, and the average diameter of the nanofibers decreased from $262 \pm 70 \mathrm{~nm}$ to $235 \pm 58 \mathrm{~nm}$ (figure 3). The fact that the average fiber diameter decreases more for the fibers with lower PEO content shows that in this case lager amounts of PEO are located at the Formatada: Não Realce surface of the fibers than in their interior. Therefore, a complete random distribution of PLLA and PEO all along the fiber is not observed. Due to the semimiscible nature of the polymers [9], for larger PLLA contents, phase separation is more provable to occur and due to the lower relative density of the PEO in the solution it is drag to the fiber surface. This process cannot take place completely before solvent evaporation in the PLLA-PEO solution with $50 \%$ of each phase, where a more random polymer distribution all along the fibers are obtained, with larger amounts of PEO located at the interior of the fibers. 


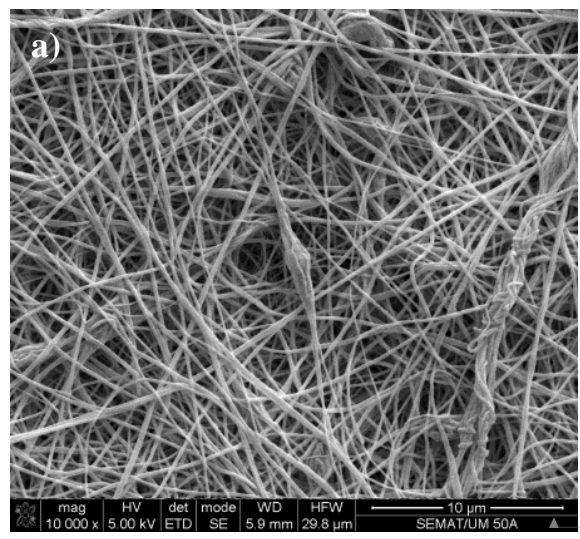

b)

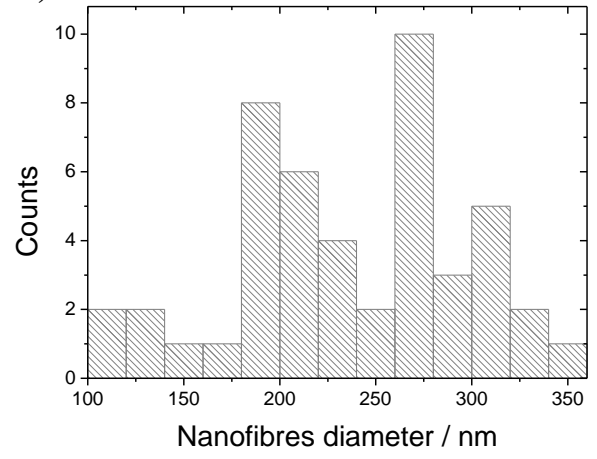

d)

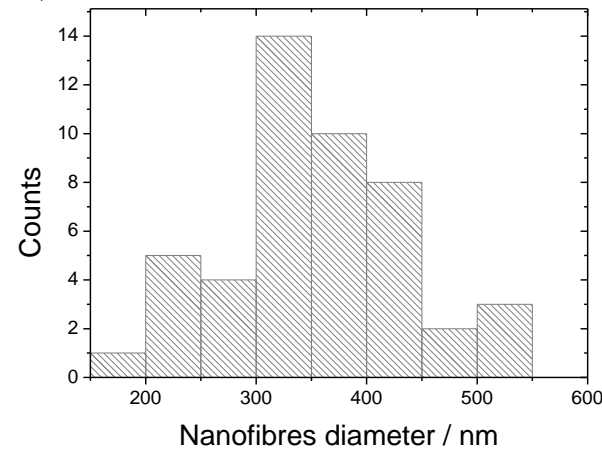

Código de campo alterado

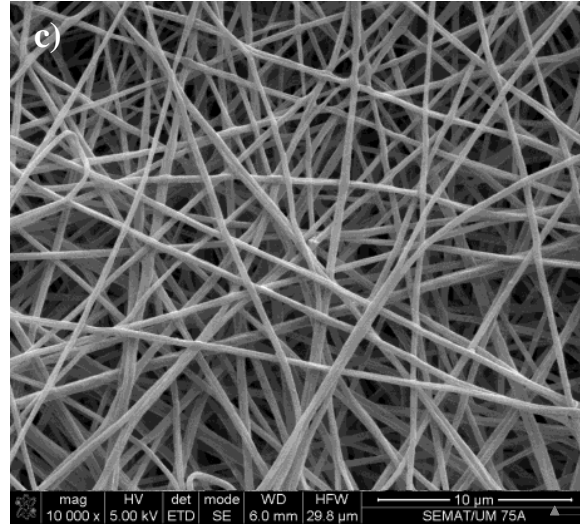

Figure 3 - Morphology of the PLLA-PEO scaffolds after PEO removal: a) SEM image of the sample with 50\% of PLLA, b) Fibre distribution for the 50/50 PLLA-PEO membrane, c) SEM image of the membrane with $75 \%$ PLLA, d) Fibre distribution for the $75 / 25$ PLLA-PEO membrane.

In order to understand the influence of the PEO on the PLLA electrospun fibrils, the topography of the samples was characterized by atomic force microscopy (AFM) (Figure 4). It can be observed that the electrospun fibres of the PLLA-PEO blend are quite smooth (figure 4a), but on the other hand, after PEO removal, the presence of higher roughness and porosity indicates that when processed, PLLA and PEO crystallizes along the nanofiber in a random distribution, and after water dissolution of the PEO polymer from the membrane, the volume occupied by this one gives rise to small pores distributed along the fiber structure, resulting therefore in a higher roughness of the electrospun PLLA remaining fibers.

\begin{tabular}{l} 
Formatada: Não Realce \\
Formatada: Não Realce \\
\hline Formatada: Não Realce \\
\hline
\end{tabular}


a)

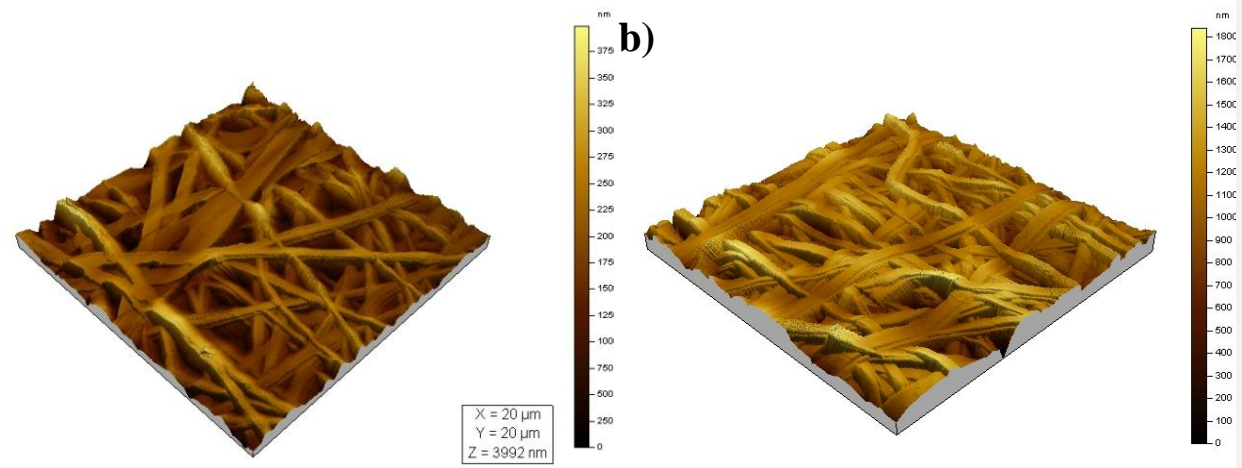

Figure 4 - AFM topographic surface images of the PLLA-PEO (50/50) sample a) before PEO removal and b) after PEO removal in water for $24 \mathrm{~h}$.

Finally, the porosity of the membranes was calculated according to equation 1 and the results are presented in table 1.

Table 1 - Porosity of the PLLA-PEO samples for different PEO contents before and after PEO removal.

\begin{tabular}{|c|c|c|}
\hline PEO Amount & $\begin{array}{c}\text { Porosity before } \\
\text { PEO Removal }\end{array}$ & $\begin{array}{c}\text { Porosity After } \\
\text { PEO Removal }\end{array}$ \\
\hline$\%$ & $\%$ & $\%$ \\
\hline 0 & $66 \pm 3$ & - \\
\hline 25 & $70 \pm 3$ & $75 \pm 3$ \\
\hline 50 & $71 \pm 3$ & $79 \pm 4$ \\
\hline
\end{tabular}

The electrospun mats of the PLLA with PEO have similar porosity than the one found for the pure PLLA polymer membrane. Just a slight increase of the porosity is observed for the PLLA-PEO membranes, when the PEO was removed. These facts support that, despite larger amounts of PEO are at the surface of the fibres for the PLLA-PEO 75/25 samples, as the fibre average diameter decreases more than in the 50/50 membranes, basically a random distribution of PEO and PLLA along the fibres is observed. Other ways, larger diminution of the fibre diameter and increase of the fibre porosity will occur by removing the relatively large quantity of PEO material from the membranes. 


\subsection{Thermal Behavior}

Figure 5 shows the thermo-gravimetric analysis for the pure PLLA, PEO and blends. For the pure materials, as expected, one single degradation process was observed. The degradation of PEO occurs at higher temperatures when compared to pure PLLA. In the case of the polymer blends, two main degradation processes were observed. The first one was due to the PLLA and the second one to the PEO thermal degradation processes, respectively (Figure 5). It was also observed that the blends have less thermal stability than the pure PLLA and the $T_{\text {onset }}$ of the blends occurs at lowest temperatures when compared to the pure polymers. It is also noticed that the increase of the PEO content in the polymer blend decreases the $T_{\text {onset }}$ of the blend (figure 6).

a)

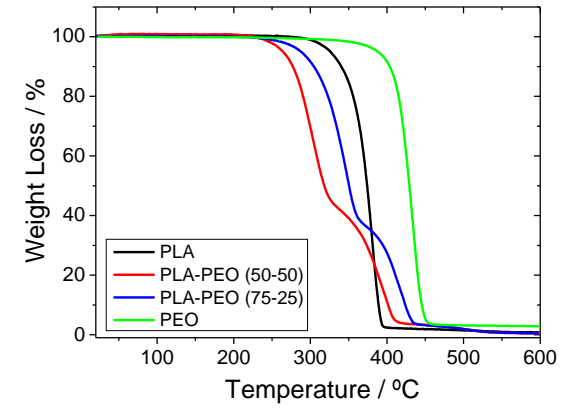

b)

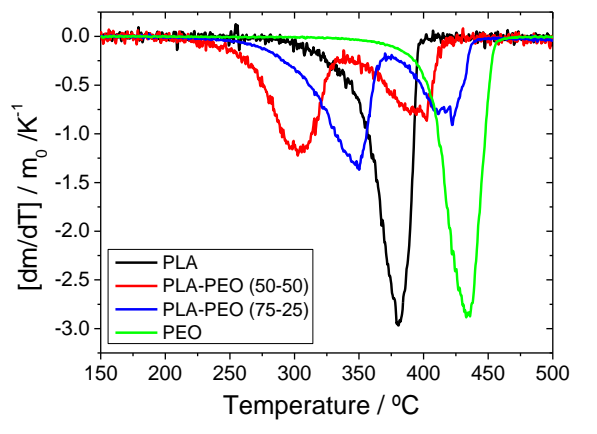

Figure 5 - a) TGA data for pure PLLA and PEO samples, as well as for the PLLA-PEO and b) DTG curves for the obtained scaffolds.

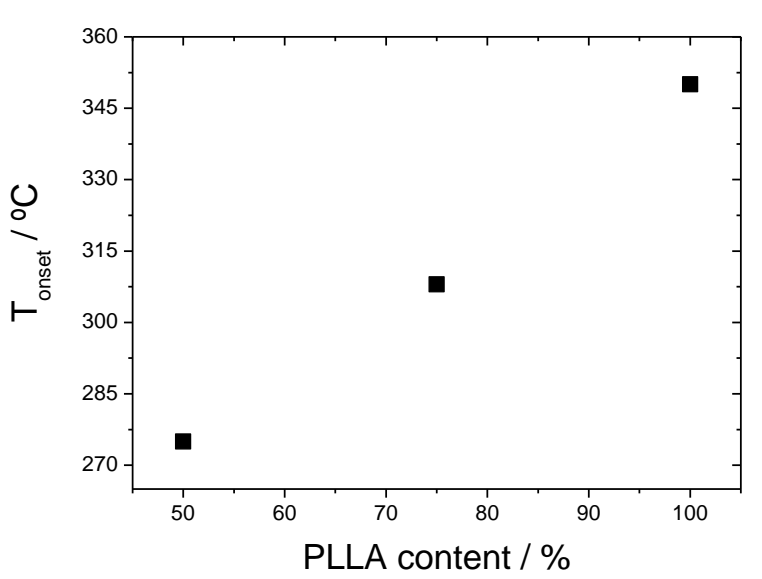

Figure 6 - Evolution of the $T_{\text {onset }}$ for the PLLA-PEO blends. 
The jet instability and deformation with fast solidification during the electrospinning process often results in a meta-stable phase [23]. Since PLLA is a slowly crystallizing polymer and its glass transition temperature is above room temperature, the samples collected at room temperature maintain a stable crystalline fraction. As mentioned in the literature [24-25], PLLA electrospun fibers from solution usually exhibits a coldcrystallization peak in DSC heating scans performed at $10{ }^{\circ} \mathrm{C} \cdot \mathrm{min}^{-1}$. Figure 7 shows the first run of the DSC heating scans performed on electrospun PLLA, PEO as well as for the electrospun blends. a)

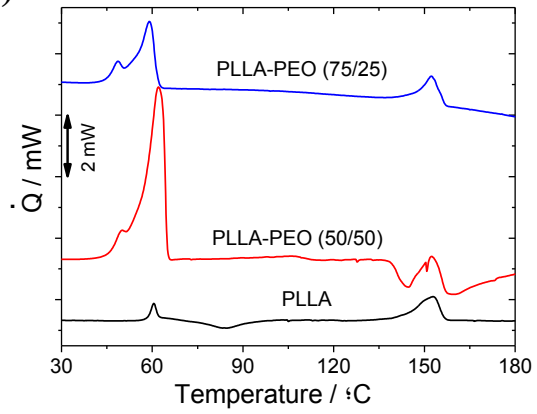

b)

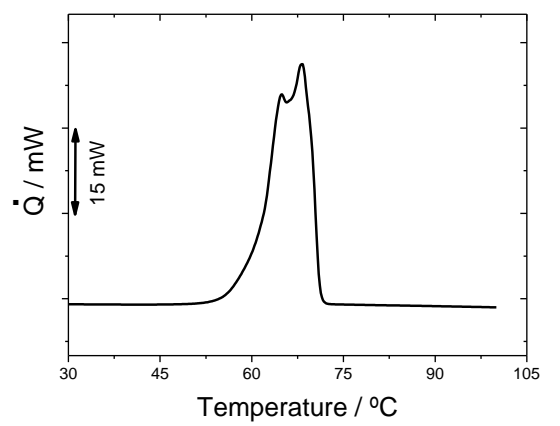

Código de campo alterado

Código de campo alterado

Formatada: Não Realce
Código de campo alterado

Figure 7 - DSC normalized thermograms of: a) electrospun PLLA and blend samples and $b$ ) first heating scan of the pure PEO mesh.

In figure 7 , the endothermic peak of PEO appears at about $56{ }^{\circ} \mathrm{C}$. One method to evaluate the miscibility of the polymers in the blend is the measurement of the variation of $T_{g}$ with the change of the weight fraction of the blend. The glass transition temperature in the miscible amorphous polymer blend changes continuously between the $T_{g}$ temperatures of the polymer components. In the case of the immiscible pair of blends, two glass transition temperatures appear separately due to the mixed polymers, and they not change with the weight fraction [26]. In this polymer blend, the $T_{g}$ of the

Formatada: Não Realce

Código de campo alterado PLLA appears in the same temperature range and the variations of the glass transition temperature of the PLLA with the weight faction is very difficult to detect, as it can be observed in figure $7 \mathrm{a}$.

$T_{g}$ of the PEO appears at about $-60^{\circ} \mathrm{C}$ and the changes in the $T_{g}$ of the PEO should be detected. Nakafuku [9] showed that the $T_{g}$ of the PEO increase slightly with the decreasing of the weight fraction of the PEO, but the increasing rate was very small, 
much smaller than the value that could be predicted using Fox equation. The authors concluded that PLLA and PEO molecules are semimiscible [9].

The melting peak of the PLLA and PEO appears in the blends but the re-crystallization peak of the PLLA only is clearly observed in the PLLA-PEO (50/50) blend during heating. The temperature corresponding to the peak of the melting of the PLLA in the blends is quite similar to the one observed to the pure PLLA.

In pure PLLA, a large overshot can be observed in the region of the glass transition, around $54{ }^{\circ} \mathrm{C}$. This endothermic peak can be ascribed to the recovering of enthalpy of the sample stored at room temperature, around $30^{\circ} \mathrm{C}$ below $T_{g}$ and thus subjected to physical ageing. The effect is also observed in the blends by a small peak that appears at low temperature just before the melting temperature of the PEO and is more pronounced for the blend with $75 \%$ PLLA. The melting temperature of the PEO in the blends slightly increases with increasing amount of PEO present in the sample. Some authors attribute this effect to morphological effects (lamellar size effects) [9]. They point that the crystallization of the PEO is hindered by the PLLA molecule, because it is possible that the PEO crystallize between the lamellae of the crystallized PLLA.

\subsection{Infrared spectra}

Infrared measurements do not reveal any new vibrational modes or significant shifts of the peak frequencies with respect to the characteristic absorption bands of the pure components of the blends, what still supports the phase separation of the two polymers in the blend.

FTIR spectra show that the PLLA-PEO membranes are composed by almost amorphous PLLA nanofibers and by high crystalline PEO electrospun nanofibers, in good agreement with DSC results and the morphology variations of the fibers after PEO removal.

The absence of the absorption band at $921 \mathrm{~cm}^{-1}$ in pure PLLA reveals that the polymer crystallizes into $\alpha$-crystals with the distorted $10_{3}$ helix conformation from solution or melt. Kister et al. reported that an absorption band at $921 \mathrm{~cm}^{-1}$ is characteristic of the $\alpha$ crystals [27]. Kang et al. assigned this vibrational band associated to the transition moment perpendicular to the chain axis to the $\mathrm{CH}_{3}$ rocking mode combined with a minor contribution from the $\mathrm{C}-\mathrm{COO}$ and $\mathrm{O}-\mathrm{CH}$ stretching modes of the $\alpha$-crystals [28]. In our membranes there is a lack of the characteristic band of the PLLA $\beta$ crystal at 908
Código de campo alterado

Formatada: Não Realce

Código de campo alterado

Formatada: Não Realce

Formatada: Não Realce

Código de campo alterado

Código de campo alterado

Formatada: Não Realce 
$\mathrm{cm}^{-1}$ [29-30], which reveals the nonexistence of this kind of crystalline structure neither in the samples as obtained by electrospinning of the pure PLLA nor in the PLLA-PEO electrospun blends (Figure 8).

After removal of the PEO polymer from the blend by immersing the scaffold in water for $24 \mathrm{~h}$ the infrared spectra of the sample only present the characteristic absorption bands of the PLLA, without any distinctive absorption band of the PEO polymer, which is in agreement with the thermal results, showing the poor miscibility of the PLLA and PEO and that the PEO is completely removed from the samples.

Formatada: Não Realce

Código de campo alterado

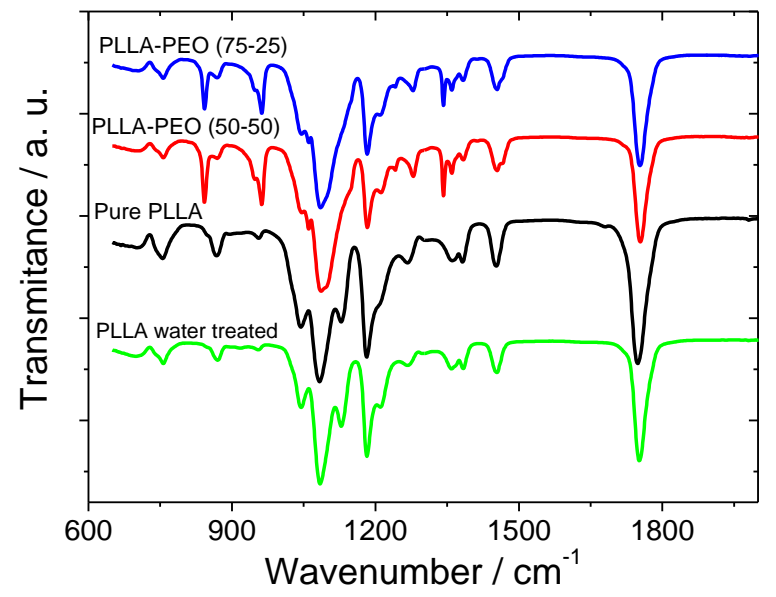

Figure 8 - Detail of FTIR spectra in the $650-2000 \mathrm{~cm}^{-1}$ region for the pure PLLA and for the PLLA-PEO blend membranes.

\section{Conclusions}

Electrospinning of PLLA - PEO blends can be achieved from the solution of both polymers in a common solvent: a mixture of MC and DMF. In this way, electrospun Formatada: Não Realce membranes can be prepared with tailored fiber diametre from some micrometers for pure PLLA membranes to few hundreds of nanometers by electrospinning of PLLAPEO solution. The mean diameter of the fibrils in the electrospun mat decreases with increasing PEO content in the blend, which also broadens the diameter distribution. This fact is due both the larger interaction of PEO with the electric field, dragging also the PLLA material, and to the reduction of the solution viscosity with increasing PEO content. The PLLA-PEO membranes are composed by almost amorphous PLLA

\begin{tabular}{|l|}
\hline Formatada: Não Realce \\
\hline Formatada: Não Realce \\
\hline Formatada: Não Realce \\
\hline
\end{tabular}


nanofibers and by high crystalline PEO electrospun nanofibers. PEO extraction from the electrospun blend is complete. This extraction decreases slightly the mean diameter of the fibers (from $260 \mathrm{~nm}$ to $235 \mathrm{~nm}$ for the PLLA-PEO 50/50 membrane) and increases membrane porosity (from $71 \%$ to $75 \%$ for the PLLA-PEO 50/50 membrane). These facts indicate that PEO and PLLA are phase separated and nearly randomly distributed along the nanofibers.

\section{Acknowledgements}

The authors thank the Portuguese Foundation for Science and Technology (FCT) Grants PTDC/CTM/73030/2006, PTDC/CTM/69316/2006 and NANO/NMedSD/0156/2007. V.S. thanks the FCT for the SFRH/BPD/63148/2009 grant. C. R. thanks the IINL for a PhD grant. JLGR acknowledge the support of the Spanish Ministry of Education through project No. MAT2010-21611-C03-01 (including the FEDER financial support) and founding in the Centro de Investigación Principe Felipe in the field of Regenerative Medicine through the collaboration agreement from the Conselleria de Sanidad (Generalitat Valenciana), and the Instituto de Salud Carlos III (Ministry of Science and Innovation). The authors also thank CeNTI - Centre for Nanotechnology and Smart Materials- for allowing the use of the electrospinning set-up and C.M. Costa from CeNTI for the high quality AFM images.
Formatada: Não Realce

Formatada: Não Realce

Formatada: Não Realce

Formatada: Não Realce 


\section{References}

1. S. Ramakrishna, et al., An introduction to electrospinning and nanofibers. Singapore: World Scientific Publishing Co. Pte. Ltd (2005).

2. S. H. Tan, R. Inai, M. Kotaki, and S. Ramakrishna, Polymer 46, 6128 (2005).

3. A. Formhals, US Patent 1975504 (1934).

4. J. Venugopal, Y. Z. Zhang, and S. Ramakrishna, J. nanoeng. nanosyst. 218, 35 (2005).

5. S. G. Kumbar, S. P. Nukavarapu, R. James, M. V. Hogan, and C. T. Laurencin, Recent Patents Biomed. Eng. 1, 68 (2008).

6. H. Pan, L. Li, L. Hu, and X. Cui, Polymer 47, 4901 (2006).

7. M. Spasova, D. Paneva, N. Manolova, P. Radenkov, and I. Rashkov, Macromol. Biosci. 8, 153 (2008).

8. H. Younes, and D. Cohn, Eur. Polym. J, 24, 765 (1988)

9. C. Nakafuku, and M. Sakoda, Polym. J. 25, 909 (1993).

10. X. Lu, C. Wang, and Y. Wei, Small 5, 2349 (2009).

11. H. Tsuji, M. Nakano, M. Hashimoto, K. Takashima, S. Katsura, and A. Mizuno, Biomacromolecules 7, 3316 (2006).

12. W. Tomaszewski, A. Duda, M. Szadkowski, J. Libiszowski, and D. Ciechanska, Macromol. Symp. 272, 70 (2008).

13. S. - Y. Gu, and J. Ren, Macromol. Mater Eng. 290, 1097 (2005).

14. J. Zeng, H. Hou, A. Schaper, J. H. Wendorff, and A. Greiner, e-Polymers 009, 1 (2003).

15. X. Xu, X. Zhuang, X. Chen, X. Wang, L. Yang, and X. Jing, Macromol. Rapid Comm. 27, 1637 (2006).

16. M. Wei, B. Kang, C. Sung, and J. Mead, Macromol. Mat. Eng, 291, 1307 (2006).

17. M. Spasova, O. Stoilova, N. Manolova, I. Rashkov, and G. Altankov, J. Bioact. Compat. Pol. 22, 62 (2007).

18. C. Nakafuku, and M. Sakoda, Polym. J. 25, 909 (1993).

19. http://rsbweb.nih.gov/ij/index.html, Image J. (2009).

20. M. Bognitzki, W. Czado, T. Frese, A. Schaper, M. Hellwig, M. Steinhart, A. Greiner, and J. H. Wendorff, Adv. Mater. 13, 70 (2001).

21. C. Ribeiro, V. Sencadas, J. L. Gómez Ribelles and S. Lanceros-Méndez, Sci Technol. Adv. Mat., acepted (2011).

22. Z. -M. Huang, Y. -Z. Zhang, M. Kotaki, and S. Ramakrishna, Compos. Sci. Technol. 63, 2223 (2003).

23. H. Zhou, T.B. Green, and Y.L. Joo, Polymer 47, 7497 (2006).

24. J. Zeng, X. Chen, Q. Liang, X. Xu, and X. Jing, Macromol. Biosci. 4, 1118 (2004).

25. R. Inai, M. Kotaki, and S. Ramakrishna, J. Polym. Sci. B: Polym. Phys. 43, 3205 (2005).

26. D. R. Paul, Polymer Blends. New York: Academic Press, 1 (1978).

27. G. Kister, G. Cassanas, and M. Vert, Polymer 39, 267 (1998).

28. S. Kang, and S. L. Hsu, Macromolecules 34, 4542 (2001).

29. J. Mijovic, and J.-W. Sy, Macromolecules 35, 6370 (2002).

30. H. Urayama, S.-I. Moon, and Y. Kimura, Macromol. Mat.Eng. 288, 137 (2003). 